\title{
The safety and feasibility of diagnostic acoustic radiation force impulse elastography used for eyes. A preliminary in vivo study
}

\author{
Li Zha ${ }^{1 *}$, Ke-Qi Chen ${ }^{2 *}$, MS, Xiao-Zhi Zheng², Jing $\mathrm{Wu}^{2}$
}

${ }^{1}$ Department of Ophtalmology, The First Affiliated Hospital of Kunming Medical University, Kunming, Yunnan Province, ${ }^{2}$ Department of Ultrasound, Yancheng Institute of Clinical (The First People's Hospital of Yancheng), Xuzhou Medical University, Yancheng, Jiangsu Province, P.R. China.

* the authors shared the first authorship

\begin{abstract}
Aims: To assess the safety and feasibility of acoustic radiation force impulse (ARFI) elastography in the eyes of rats. Material and methods: One hundred and twenty-six SD rats were divided into 7 groups. Group 1 was the control group and Group 2 to Group 7 were investigated by ARFI elastography using increased powers of 5\%, 10\%, 20\%, 50\%, and $100 \%$ and repetitions of 5 or 10 . The changes of ocular architecture structures and functions were examined by ophthalmoscopic, histopathologic, and light reflex examination, and the expression level of IL-6, IL-8, and TNF- $\alpha$ was determined in days 1, 3, and 7 after ARFI elastography, respectively. Results: A satisfactory ARFI elastography was obtained in all SD rats. The shear wave velocity (SWV) values of soft tissues behind the eyeball were significantly greater than those of the eyes $(1.89 \pm 0.95 \mathrm{vs}$. $0.84 \pm 0.36 \mathrm{~m} / \mathrm{s}, \mathrm{p}<0.05)$. The SWV values of eyes and the soft tissues behind the eyeball did not differ among any groups at any time-point $(\mathrm{p}>0.05)$. The SD rats of each group showed good light perception, pupillary light reflex, and avoidance reaction induced by the sudden bright light from the dark environment at any time. In each group, ocular architecture structures were well preserved, and with a normal expression level of IL-6, IL-8, and TNF- $\alpha$ ( $p>0.05)$. Conclusions: Diagnostic ARFI elastography is a safe and feasible ultrasonic imaging mode and may potentially be applied for human eyes in its present form.

Keywords: safety; ultrasound elastography; acoustic radiation force impulse; shear wave velocity; eye.
\end{abstract}

\section{Introduction}

Ultrasound elastography (strain or shear wave type) has been widely used in the clinical setting for the diagnosis of benign and malignant tumors in the thyroid $[1,2]$, breast [3,4], liver [5-8], kidney [9], prostate [10,11] and lymph nodes $[12,13]$, for guiding and monitoring

Received 17.08.2016 Accepted 04.01.2017

Med Ultrason

2017, Vol. 19, No 2, 185-189

Corresponding author: Xiaozhi Zheng

Department of Ultrasound, Yancheng Institute

of Clinical Medicine (The First People's

Hospital of Yancheng)

Xuzhou Medical University

166 West Yulong Road, Yancheng 224005,

Jiangsu Province, P.R. China

Phone:+86 051588508717

Fax: +860515 88500299

E-mail: zxzycyy1232@126.com the interventional diagnosis and treatment [14,15], and even for the assessment of the placenta $[16,17]$ and fetal maturity [18]. In ophthalmology the mechanical properties of cornea [19], lens [20], and eye [21,22] have also been studied using elastography. The ocular tissues are particularly susceptible to mechanical and thermal injury associated with excessive ultrasound energy, so that the Food and Drug Administration (FDA) and World Federation for Ultrasound in Medicine and Biology have imposed strict thermal index (TI) and mechanical index (MI) limits for diagnostic ocular ultrasound applications (TI <1.0, MI <0.23) [23]. The safety and feasibility of ultrasound elastography used for eyes remains unknown.

The present study aims to assess the safety and feasibility of diagnostic acoustic radiation force impulse (ARFI) elastography used for eyes in the living rat by employing a diagnostic ARFI elastography at a fixed high ultrasound pressure (mechanical index=1.6). 


\section{Material and methods}

This experimental study was performed in the central laboratory of our hospital from Mar 2, 2016 to Sep 25, 2016, with the approval of the Ethics Committee of our hospital.

\section{Animal preparation}

In this experiment, 126 Sprague-Dawley (SD) rats (male or female; age, 8 10 weeks; weight=180 200g; SLACCAS, Shanghai, China) were enrolled. The animals were bred, maintained, and sacrificed humanely in strict compliance with the policies stated in the statement of Association for Research in Vision and Ophthalmology for the use of animals in ophthalmic and vision research.

\section{Imaging techniques}

ARFI elastography was performed using Siemens ACUSON S2000 ultrasound system (Siemens, Erlangen, Germany), with a linear array transducer (9L4), harmonic imaging $(8 \mathrm{MHz})$, a fixed mechanical index of 1.6 and a fixed duration of $1 \mathrm{~ms}$. A "pushing" beam is transmitted through the target region of interest where produces localized displacements. The wave propagation speed in that region of interest is automatically measured by the system and a numerical value (in $\mathrm{m} / \mathrm{s}$ ) is then reported.

\section{Protocol of the study}

The rats were divided into 7 groups $(n=18)$ : Group 1 -the control group and the Group $2-7$ the experimental groups, receiving ARFI elastography with different powers and repetitions (Group 2: power 5\%, repetitions 5; Group 3: power 10\%, repetitions 5; Group 4: power 20\%, repetitions 5; Group 5: power 20\%, repetitions 10; Group 6 : power $50 \%$, repetitions 10 ; Group 7 : power $100 \%$, repetitions of 10).

\section{Eye ARFI elastography}

The SD rats were well anesthetized by an intraperitoneal injection of $10 \%$ chloral hydrate $(350 \mathrm{mg} / \mathrm{kg}$ body weight). The 9L4 transducer was placed directly onto the conjunctival surface, after smearing a small amount of coupling medium. Then, ARFI elastography was performed with the preliminary identification of a target region of interest (box with a fixed dimension of $6 \times 5 \mathrm{~mm}$ ) on an accurate conventional ultrasound image (fig 1). Six measurements of the shear wave velocity (SWV propagating in the eyes and the soft tissues behind the eyeball) were performed, respectively. Finally, the average SWV values of each part were obtained.

\section{Light reflex examination}

Five eyes of SD rats of each group were selected randomly on days 1,3 , and 7 after ARFI elastography. The light reflex, including light perception, pupillary light reflex, and the avoidance reaction induced by the sudden

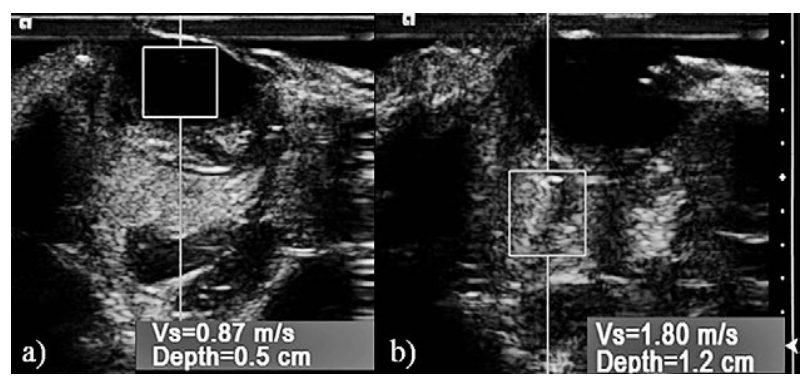

Fig 1. Shear wave velocity measurement at vitreous body (a) and the soft tissue behind the eyeball (b) using acoustic radiation force impulse elastography.

bright light from the dark environment, was examined by two masked observers.

\section{Ophthalmoscopic examination}

Five eyes of SD rats of each group were selected randomly and ophthalmoscopically examined by two masked observers on days 1, 3, and 7 after ARFI elastography. Fundus photographs were taken using a surgical microscope (SM-2000 J; Eder).

\section{Histopathologic examination}

On days 1, 3, and 7 after ARFI elastography, the SD rats (three per time-point) were sacrificed with a fatal dose of $10 \%$ chloral hydrate. The eyes were enucleated, and fixed in $10 \%$ formaldehyde solution at a room temperature. Thereafter, they were embedded in paraffin, and cut into $5 \mu \mathrm{m}$-thick sections. Subsequently, the sections were stained with hematoxylin-eosin to observe ocular architecture, inflammatory cell infiltration, and proliferation response using light microscopy (Zeiss Axiovert $\mathrm{S}$ 100, Jena, Germany). All the results were confirmed by two blinded experts with experience in ophthalmological pathology.

\section{Enzyme-linked immunosorbent assay}

On days 1, 3 and 7 after ARFI elastography, the SD rats (three per time-point) were sacrificed with an overdose of $10 \%$ chloral hydrate. The eyes were extracted and grinded into homogenate. IL-6, IL-8 and TNF- $\alpha$ were measured using a capture sandwich kit with biotinylated affinity purified mouse monoclonal antibodies to rat IL-6, IL-8 and TNF- $\alpha$ (Senxiong, Shanghai, China). Briefly, a flat-bottom ELISA plate (Costar 96-well) was coated with mouse anti-rat IL-6, IL- 8 and TNF- $\alpha$ antibody, $100 \mu \mathrm{L}$ of standard preparation (or sample) was added in the wells and incubated at $37^{\circ} \mathrm{C}$ for two hours. After washing six times, $100 \mu \mathrm{L}$ of biotinylated mouse anti-rat IL-6, IL- 8 and TNF- $\alpha$ was added and incubated at $37^{\circ} \mathrm{C}$ in the dark for one hour, washed, and $100 \mu \mathrm{L}$ of horseradish peroxidase labeled streptavidin was added and incubated at $37^{\circ} \mathrm{C}$ for one hour. The wells were washed six times again, and incubated with $100 \mu \mathrm{L}$ of 
substrate solution for 5-10 minutes. Finally, $50 \mu \mathrm{L}$ of stop buffer was added to each well. Absorbance at $492 \mathrm{~nm}$ was measured using a microplate reader (Molecular Devices, Sunnyvale, USA).

\section{Statistical analysis}

Data from ARFI elastography were compared using an unpaired Student's t-test. Data from ELISA are presented as the mean \pm standard deviation (SD). One-way analysis of variance (One-way ANOVA) was used to determine the significance of the difference in a multiple comparison. A value of $\mathrm{p}<0.05$ was the considered statistically significant. SPSS, version 13 (SPSS Inc., Chicago, IL, USA) was used for the statistical analysis.

\section{Results}

\section{Eye ARFI elastography}

A satisfactory ARFI elastography was obtained in all $\mathrm{SD}$ rats. The mean SWV value was $0.84 \pm 0.36 \mathrm{~m} / \mathrm{s}$ (in the range of 0.52 to $2.13 \mathrm{~m} / \mathrm{s}$ ). The values did not differ among groups at any time-point, $\mathrm{p}>0.05$ ). The mean $\mathrm{SWV}$ value of the soft tissues behind the eyeball was $1.89 \pm 0.95$ $\mathrm{m} / \mathrm{s}$ (in the range of 0.64 to $4.80 \mathrm{~m} / \mathrm{s}$ ) without difference between any groups at any time-point ( $>0.05)$, too. In addition, we found that SWV values of the soft tissues behind the eyeball were significantly greater than those of eyes among any groups at any time-point $(\mathrm{p}<0.05)$.

\section{Light reflex examination}

All the SD rats showed good light perception, pupillary light reflex, and the avoidance reaction induced by the sudden bright light from the dark environment. There was no difference among any groups at any time-point, whether according to the light perception and pupillary light reflex or according to the avoidance reaction induced by the sudden bright light from the dark environment ( $\mathrm{p}>0.05)$.

Ophthalmoscopic and histopathologic examination

As shown in figure 2, all the SD rats of any groups at any time-point showed a normal ocular architecture, without edema, opacity of optical system (corneas, aqueous fluid, lens and vitreous bodies), retinal folds, retinal detachments, inflammatory cell infiltration and proliferative membrane formation. The eye fundus was clearly visible.

\section{Enzyme-linked immunosorbent assay}

Compared to Group 1, each experimental group (from Group 2 to Group 7) at any time-point did not show a higher expression level of IL-6, IL- 8 , and TNF- $\alpha$ $(p>0.05)$. Moreover, the values did not differ to each other at any time-point ( $>0.05)$. The mean values of IL-6, IL8 , and TNF- $\alpha$ from Group 1 to Group 7 at any time-point was about $44.92 \pm 7.51,77.89 \pm 16.25$, and $64.59 \pm 7.88 \mathrm{pg} /$ $\mathrm{ml}$, respectively.

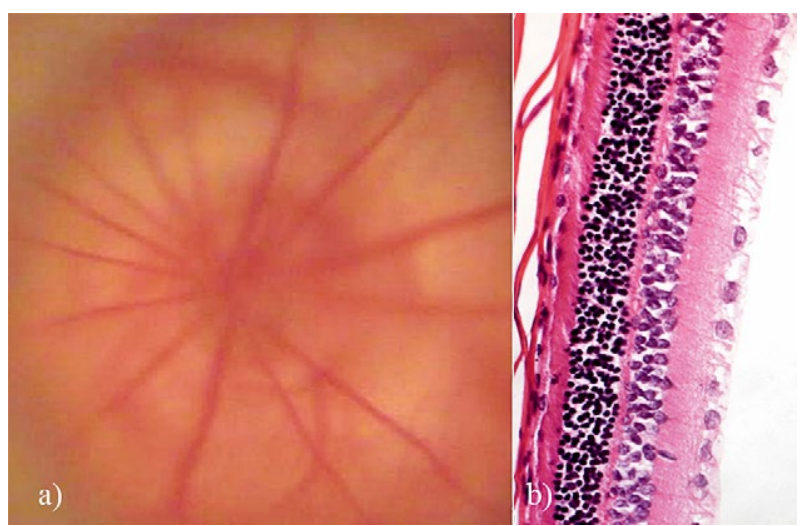

Fig 2. a) Ophthalmoscopic examination of the eye fundus. Magnification $\times 25$; b) Results of the histopathologic examination of eye. Magnification $\times 400$.

\section{Discussions}

In recent years the eye elastic properties have been studied using ultrasound elastography (strain or shear wave type), such as corneal elasticity in an ex-vivo porcine eye model [19], iridociliary and lenticular elasticity in an ex-vivo rabbit eye model [20], or vitreous cavity elastic properties in human's blind eyes [21]. The authors pointed out that this modality may potentially be applied in human eyes. However, unlike common ultrasound, whose safety has been established, the investigation of eye pathology using ARFI elastography is still on debate.

In our study, we maintained at a fixed value, the mechanical index and the duration and harmonic imaging frequency, and we adjusted only the power and repetitions. It is worth noting that a fixed mechanical index of 1.6 means a very high ultrasound pressure. Surprisingly, we noticed that even a condition of power of $100 \%$, mechanical index of 1.6 and a repetition of 10 did not cause damage to the eyes. In each group, ocular architecture structures and functions were all well preserved, and there was no obvious inflammation, too. These results laid a foundation for the application of this technology in clinical ophthalmology. This also makes us reflect on our previous studies in which we had confirmed that a condition of therapeutic ultrasound irradiation (frequency, 1 $\mathrm{MHz}$, power, $1-3 \mathrm{~W} / \mathrm{cm}^{2}$, duty cycle, $50 \%$, pulse recurrent frequency, $100 \mathrm{~Hz}$, duration, $300 \mathrm{~s}$ ) did not cause damage to the eyes $[22,24]$. Although the ultrasound parameters in these studies can not be directly compared, they still have a reference value for the safety of diagnostic ARFI elastography used for eyes. We speculate that ARFI elastography did not cause damage to the eyes because it was a very short duration $(1 \mathrm{~ms})$ even if under 
a conditions of a great power $(100 \%)$ and a very high pressure (mechanical index of 1.6).

We also noticed that the average SWV values of the soft tissues behind the eyeball were about twice as great as those of the eyes among any groups at any time-point, which indicates the eyes and soft tissues behind the eyeball had significantly different elastic properties. These results could help in understanding the bio-mechanical behavior of ocular tissues and relative diseases, such as intra-ocular and extra-ocular tumors, vitreous opacity, retinal detachment, intraocular new membrane formation, optic neuritis after the eyeball, high intraocular pressure, high intracranial pressure, etc.

The main limitation of the present study is that we did not examine the shape and structure changes of the eye using electron microscopy, neither did we test other inflammatory factors so that maybe some eye damage was not detected. Another limitation of the present study is that the box dimension of the region of interest was fixed and large; the SWV value was the average of multiple parts in the region of interest. Thirdly the ultrasonic parameters of activated ARFI elastography could not be adjusted. The fourth limitation is that we did not follow-up the long-term potential damages of ARFI elastography to rat eyes, although this damage may be self-healing. Finally, although the rat eye model has been extensively used in experimental studies, it is also known to present distinct anatomical and physiological differences compared with the human eyes.

Our ongoing work is focused on upgrading the setup for in vivo eye experiments to further evaluate the longterm side effects of diagnostic ARFI elastography, as well as evaluating the safety of other ultrasound elastography modes, such as real-time two-dimensional SWE.

\section{Conclusion}

This is the first study on the safety and feasibility of diagnostic ARFI elastography in a rat eye model. We found that diagnostic ARFI elastography is a safe ultrasonic imaging mode, instead of real-time two-dimensional SWE, and may be potentially applied in human eyes in its present form due to a very short duration and artificially controllability.

Acknowledgements: The authors gratefully acknowledge the technical assistance and helpful discussions of Li-Zhu Miao, You Zhou, Xiao Wang, Wen-Yan Zhou, Xiao-Qin Huang from the Department of Ultrasound, The First People's Hospital of Yancheng.

Conflict of interest: none

\section{References}

1. Hou XJ, Sun AX, Zhou XL, et al. The application of virtual touch tissue quantification (VTQ) in diagnosis of thyroid lesions: A preliminary study. Eur J Radiol 2013;82:797-801.

2. Zhang YF, Xu HX, He Y, et al. Virtual touch tissue quantification of acoustic radiation force impulse: a new ultrasound elastic imaging in the diagnosis of thyroid nodules. PLoS One 2012; 7:e49094.

3. Jin ZQ, Li XR, Zhou HL, et al. Acoustic radiation force impulse elastography of breast imaging reporting and data system category 4 breastlesions. Clin Breast Cancer 2012;12:420-427.

4. Bai M, Du L, Gu J, Li F, Jia X. Virtual touch tissue quantification using acoustic radiation force impulse technology: initial clinical experience with solid breast masses. J Ultrasound Med 2012;31:289-294.

5. Gallotti A, D’Onofrio M, Pozzi Mucelli R. Acoustic Radiation Force Impulse (ARFI) technique in ultrasound with Virtual Touch tissue quantification of the upper abdomen. Radiol Med 2010;115:889-897.

6. Zhang P, Zhou P, Tian SM, Qian Y, Deng J, Zhang L. Application of acoustic radiation force impulse imaging for the evaluation of focal liver lesion elasticity. Hepatobiliary Pancreat Dis Int 2013;12:165-170.

7. Gallotti A, D'Onofrio M, Romanini L, Cantisani V, Pozzi Mucelli R. Acoustic Radiation Force Impulse (ARFI) ultrasound imaging of solid focal liver lesions. Eur J Radiol 2012;81:451-455.

8. Monti L, Manco M, Lo Zupone C, et al. Acoustic radiation force impulse (ARFI) imaging with Virtual Touch Tissue Quantification in liver disease associated with cystic fibrosis in children. Radiol Med 2012;117:1408-1418.

9. Stock KF, Klein BS, Vo Cong MT, et al. ARFI-based tissue elasticity quantification in comparison to histology for the diagnosis of renal transplant fibrosis. Clin Hemorheol Microcirc 2010;46:139-148.

10. Zheng XZ, Ji P, Mao HW, et al. A novel approach to assessing changes in prostate stiffness with age using virtual touch tissue quantification. J Ultrasound Med 2011;30:387390.

11. Zheng X, Ji P, Mao H, Hu J. A comparison of virtual touch tissue quantification and digital rectal examination for discrimination between prostate cancer and benign prostatic hyperplasia. Radiol Oncol 2012;46:69-74.

12. Kilic F, Velidedeoglu M, Ozturk T, et al. Ex Vivo Assessment of Sentinel Lymph Nodes in Breast Cancer Using Shear Wave Elastography. J Ultrasound Med 2016;35:271277.

13. Azizi G, Keller JM, Mayo ML, et al. Shear Wave Elastography and Cervical Lymph Nodes: Predicting Malignancy. Ultrasound Med Biol 2016;42:1273-1281.

14. Ma BY, Parajuly SS, Ying SX, Lan PY. Application of shear wave elastography in fine needle aspiration biopsy for thyroid nodule. J Pak Med Assoc 2014;64:954-957.

15. Koh J, Jung DC, Oh YT, et al. Additional targeted biopsy in clinically suspected prostate cancer: prospective ran- 
domized comparison between contrast-enhanced ultrasound and sonoelastography guidance. Ultrasound Med Biol 2015;41:2836-2841.

16. K1lıç F, Kayadibi Y, Yüksel MA, et al. Shear wave elastography of placenta: in vivo quantitation of placental elasticity in preeclampsia. Diagn Interv Radiol 2015;21:202-207.

17. Quibel T, Deloison B, Chammings F, et al. Placental elastography in a murine intrauterine growth restriction model. Prenat Diagn 2015;35:1106-1111.

18. Zheng XZ, Wu J, Tan XY. A novel approach to assessing fetal tissue stiffness using virtual touch tissue quantification. Med Ultrason 2016;18:70-74.

19. Tanter M, Touboul D, Gennisson JL, Bercoff J, Fink M. High-resolution quantitative imaging of cornea elasticity using supersonic shear imaging. IEEE Trans Med Imaging 2009;28:1881-1893.

20. Detorakis ET, Drakonaki EE, Ginis H, Karyotakis N, Pallikaris IG. Evaluation of iridociliary and lenticular elasticity using shear-wave elastography in rabbit eyes. Acta Medica (Hradec Kralove) 2014;57:9-14.
21. Detorakis ET, Drakonaki EE, Tsilimbaris MK, Pallikaris IG, Giarmenitis S. Real-time ultrasound elastographic imaging of ocular and periocular tissues: a feasibility study. Ophthalmic Surg Lasers Imaging 2010;41:135141.

22. Zheng XZ, Du LF, Wang HP, Gu Q. A novel approach to attenuate proliferative vitreoretinopathy using ultrasoundtargeted microbubble destruction and recombinant adenoassociated virus-mediated RNA interference targeting transforming growth factor- $\beta 2$ and platelet-derived growth factor-B. J Gene Med 2012;14:339-347.

23. Palte HD, Gayer S, Arrieta E, et al. Are ultrasound-guided ophthalmic blocks injurious to the eye? A comparative rabbit model study of two ultrasound devices evaluating intraorbital thermal and structural changes. Anesth Analg 2012;115:194-201.

24. Zheng XZ, Li HL, Du LF, Wang HP, Gu Q. In vivo and in vitro effects of ultrasound or/and microbubbles on recombinant adeno-associated virus-mediated transgene expression in the retina. Asian Biomed 2009;3:497-506. 